\title{
AMORPHOUSE ICE
}

\author{
Valentin M. Silonov \\ Lomonosov Moscow State University, Department of Physics, http://www.phys.msu.ru \\ 119991 Moscow, Russian Federation \\ silonov_v@mail.ru
}

\section{Vasilii V. Chubarov}

Lomonosov Moscow State University, Department of Physics, http://www.phys.msu.ru 119991 Moscow, Russian Federation

chub-rov@mail.ru

\begin{abstract}
The detailed analysis of published data was carried out for amorphous ices. It is shown that on modern representations the amorphous ice exists in different states only at the temperature below the glass transition temperature 136K. The X-ray diffraction method was used for studying of structure formation, because this method is the basic way of producing the direct information about structure of investigated object. For researching the X-ray scattering by ices near the melting point the precision low-temperature devices for X-ray diffractometer were elaborated. The improved device provides virtually unlimited in time maintaining of temperature of investigated sample in the range from $0^{\circ} \mathrm{C}$ to $-25^{\circ} \mathrm{C}$ with precision $\pm 0.1^{\circ} \mathrm{C}$. For the first time, as a result of researches, the coexistence of amorphous structure and crystalline hexagonal structure of ice was established near melting point. The one diffuse maximum was able to identify on one of the diffractional pattern of ice, obtained from distillated water, with the almost complete absence of structural reflexes. There is the sign of completely amorphous state. The radial distribution functions were calculated from obtained diffractional patterns. They are shown that near the melting point the first maximums of radial distribution function of amorphous ice close in positions to first radii of coordination spheres of hexagonal ice. The revealed splitting of first maximum of radial distribution function can be explained by increasing of interatomic distances of neighbor atoms lying on different levels. On prolonged exposure the permanent reallocation on diffractional patterns of diffuse scattering maxima was revealed on ice sample at the constant temperature $-10^{\circ} \mathrm{C}$. It is proved, that amorphous phase of obtained from distillated water ice has the metastable character.
\end{abstract}

Keywords: amorphous ice, X-ray diffuse scattering.

PACS:61.05.cp; 07.85.Nc

Bibliography - 35 references

Received 20.04.2015

RENSIT, 2015, 7(1):55-67

DOI: $10.17725 /$ rensit.2015.07.055

\section{Contents}

1. INTRODUCTION (55)

2. Amorphous ice AT LOW TEMPERATURES AND ATMOSPHERIC PRESSURE (56)

3. Amorphous ice AT LOW TEMPERATURES AND HIGH PRESSURES (59)

4. The amorphous ice near the Melting POINT AT ATMOSPHERIC PRESSURE (61)

5. Conclusions (66)

REFERENCES (66)

\section{INTRODUCTION}

Water is one of the most popular compounds in nature. Water is a unique natural object, since plays the important role in the formation of physical and chemical processes not only on Earth, but also in interstellar space. The uniqueness of water is manifested in the fact that the simple structure of water molecules is in stark contrast to the vast variety of different phases and complex phase diagrams of water. In despite of the large and growing number of scientific studies on the structure of water, so far there is no complete phase diagram of water.

The water may exist in the gaseous, liquid and solid forms. The studying of the structure of water in the gas and the liquid state has a long history. This studying was passing ahead through accessibility and visibility of the objects, as well as underdevelopment of that time the methods and means of scientific research.

Investigation of the structure of water in the solid phase, i.e. water ice, rapidly started with the advent of $X$-ray analysis method. It has become 
the most common method for determining the structure of matter in view of the simplicity and relative cheapness. Currently, the $X$-ray analysis continue to play a major role in the study of processes of structure and phase transitions of various substances and allows to obtain direct information about the structure of the object being studied. The water forms the solid phases more than any other known substance. The result of extensive researches (until 1974) of the existence of multiple forms of ice by different research groups using modern methods of the existence of multiple forms of ice is given in the beautiful encyclopedic review [1]. At present 15 different stable and metastable phases crystalline ice are known [2]. It is found that in the crystalline phase ice the oxygen atoms are in fixed positions relative to each other, and the positions of the hydrogen atoms may be disordered, but obey Bernal-Fowler rule [3]. According to this rule, each oxygen atom is covalently bonded to two hydrogen atoms and each oxygen atom in the molecule of water is linked to two other oxygen atoms by weak hydrogen bonds. Thus, a hydrogen atom is always located between each pair of oxygen atoms, and a regular grid of crystal lattice is the result. The results of studies in recent years of different crystalline forms of ice and their mutual transitions with using mainly by $X$-ray and neutron diffraction methods are discussed in detail in review [4].

However, the structure of the water allows the creation of many more unique natural forms. Thus upon cooling below $0^{\circ} \mathrm{C}$ ultrapure water without germinal centers or impurities may not crystallize in a stable phase under ice $I_{b}$ homogeneous nucleation temperature $T_{H^{*}}$ It may remain in the form of supercooled water up to a temperature of $\sim 231 \mathrm{~K}$ at normal pressure [5]. Upon further cooling down to the glass transition temperature $T_{g}$, there is a structure in the form of an amorphous solid ice, in which the crystalline and random mesh atoms coexist together. For condensed matter physics the study of such a structure is a complex task. The exact determination of the $T_{g}$ has been the subject of years of research and active debate. Currently it accepted $T_{g} \sim 136 \mathrm{~K}$, as a result of calorimetric experiments with amorphous ice at normal pressure [6].

Temperature range between $T_{h}$ and $T_{g}$ in the foreign literature is called "no man's land". In this region, the supercooled water freezes spontaneously below the temperature $T_{H}$, and amorphous ice crystallizes rapidly at temperatures above $T_{g}$. Experiments in this field are not fulfilled, because they require superfast millisecond methods of recording parameters of liquid water [7].

\section{AMORPHOUS ICE AT LOW TEMPERATURES AND ATMOSPHERIC PRESSURE}

An important and significant difference between amorphous and crystalline structures is the lack of long-range order in the amorphous structure, resembling the chaotic structure of liquid water. The emergence of the amorphous structure is determined by $X$-ray diffraction method because the lack of Bragg diffraction in the crystal lattice is a criterion amorphization of substance. This results in the absence of intense reflections from the crystal lattice and identity of diffraction pattern shown in Fig. 1 for water [8] and for the amorphous ice [9]. Depending on the method of preparation of amorphous ice under normal pressure the amorphous ices are differed in the literature on the ASW (amorphous state water), produced mainly by cooling the low-temperature water vapor on the substrates and the $H G W$ (hyperquenched glass water), resulting from rapid quenching of water. Because thermodynamic properties of these ices are practically identical, they may be regarded as one and the same phase of amorphous ice [10]. First amorphous ice was prepared under normal pressure of water vapor deposition on a cold substrate [11]. It is shown that the nature of the resulting solid sample on the substrate depends strongly on the substrate temperature. 

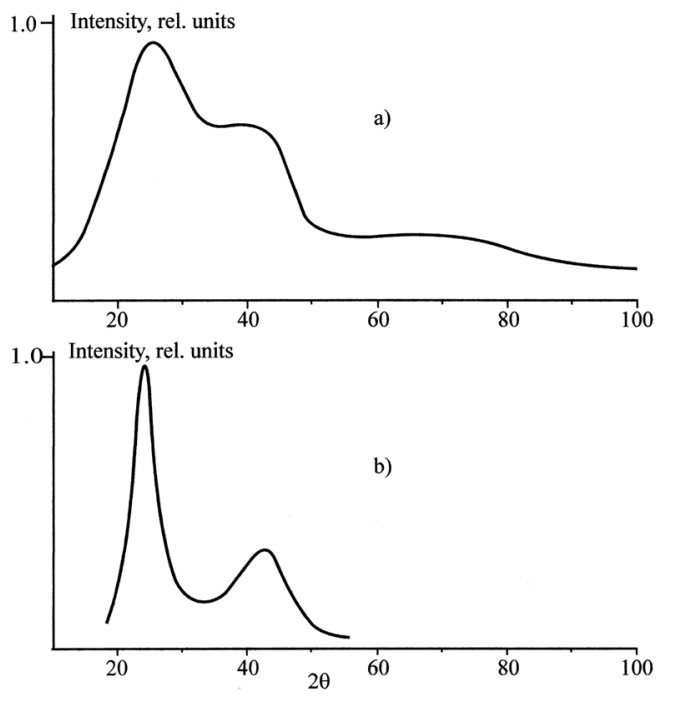

Fig. 1. The XRD patterns of a) water, registered at $1.5^{\circ} \mathrm{C}$

[8]; b) amorphous ice, registered at $-175^{\circ} \mathrm{C}$ [9].

By $X$-ray diffraction it was determined that at a substrate temperature higher than $-80^{\circ} \mathrm{C}$ ice Ih was formed, and at a temperature of $-110^{\circ} \mathrm{C}$ the two diffuse lines were appeared on $X$-ray pattern, resembling the spectrum of liquid water. From this experiment it was concluded that at a substrate temperature below $-110^{\circ} \mathrm{C}$ an amorphous material was produced. The mutual transformations $I_{b}, I_{c}$ and amorphous ice (vitrified water) when the temperature changes were reviewed in [9]. On the basis of $X$-ray diffractometer the low-temperature cell was designed with continuously variable temperature control of the sample obtained by depositing of water vapor on a cold substrate. The device provides a minimal temperature of sample $-192^{\circ} \mathrm{C}$. A typical diffraction pattern of vitrified water, which is characterized by two intense maxima, is shown on Fig.1. The first peak is located at $24^{\circ}$ on $2 \theta$ and corresponds to the Bragg Distance $3.71 \AA$, the second by $42^{\circ}$ on $2 \theta$, and corresponds to the distance $2.15 \AA$. It is noted that the distance of $3.71 \AA$ is very close to the value of $3.67 \AA$, the corresponding line (111) of cubic ice. The broadening of the lines is clearly associated with the formation of a disordered atomic grid of amorphous structure. The mixture of water and vitrified cubic ice converted irreversibly to hexagonal phase ice in the temperature range from $-160^{\circ} \mathrm{C}$ to $-130^{\circ} \mathrm{C}$. It is important to note that, according to the authors, it is impossible to obtain vitrified water directly from the liquid water. Using $X$-ray diffraction method, the authors have discovered in the sample deposited on a substrate, the coexistence of cubic ice $I_{c}$ and a hexagonal ice $I_{b}$ in admixture, which is transformed into ice $I_{b}$ at about $-75^{\circ} \mathrm{C}$ [12]. In this work the bond length $\alpha_{0}$ for ice $I_{c}$ was determined as the $6.36 \pm 0.03 \AA$ at $-130^{\circ} \mathrm{C}$. The same way $X$-ray diffraction method was used by authors [13], but the water vapor was precipitated under vacuum conditions. It is shown that the amorphous structure is converted accurately into ice $I$ in the range from $-133^{\circ} \mathrm{C}$ to $-117^{\circ} \mathrm{C}$ and ice $I$, in turn, is transformed into ice $\mathrm{Ih}$ in a wide range of temperatures, beginning from the temperature of $-73^{\circ} \mathrm{C}$. The real possibility of obtaining the solid ASW, free from the presence of crystalline ice, is demonstrated in [14]. The resulting $X$-ray patterns of film with a thickness of 50 microns on a pure copper substrate at temperatures of $50 \mathrm{~K}, 77 \mathrm{~K}$ and $145 \mathrm{~K}$ show a lack of crystal structure. However, in spite of experiments with only an amorphous structure, the authors were unable to accurately determine the temperature of transition from the $A S W$ to the ice structure $I$.

The original method of obtaining $H G V$ $(A S W)$ was proposed in [15]. The water specially prepared in cryogenic media type ethane, propane and butene-1, was passed under pressure through an injector in the form of a jet. As was shown in [16] the water can move into a vitrified state when its ultrafast cooling at a rate of about $10^{8} \mathrm{~K} / \mathrm{s}$. Carried out in [15] the calculation showed if jet diameter was $10 \mu \mathrm{m}$ and a pressure was $400 \mathrm{~atm}$, the rate of cooling water in the cryogenic medium reaches a value of $10^{10} \mathrm{~K} / \mathrm{s}$. However, $X$-ray diffraction method showed that the diffraction patterns of fractions obtained in experiments differ significantly from the diffraction patterns of $H G V$. Mainly 
fractions consist of ice Ih with admixture vitrified material such as $H G V$. Thus, in jet stream of the liquid there were or low speed cooling, forming ice $I_{b}$, or very high, leading to the formation of $H G V$. According to the authors this is due to formation of vortices in a vigorously stirred cryogenic medium.

Interesting results are presented in [16]. Obtained by deposition of water vapor the amorphous ice when heated above $T_{g}$ $(120 K-140 K)$ was transformed into a viscous liquid, at that dependences of viscosity on temperature differed significantly for this liquid and liquid water. Investigations of thin films of ice by fundamentally different methods showed that the viscous liquid coexists with the crystalline cubic ice in the temperature range $140 \mathrm{~K}-210 \mathrm{~K}$. The authors suggest that this form of water is very common in nature and exist in the subsurface layers of comets and on the surfaces of some planets and satellites.

The result of years of research of amorphous ice at normal pressure is the fact that when heated above the glass transition temperature $T_{g} A S W$ and $H G W$ transformed into a metastable cubic ice $I$. The density of this amorphous ices measured by buoyancy in the liquid nitrogen-argon mixture, determined as $0.94 \pm 0.01 \mathrm{~g} / \mathrm{cm}^{3}$ [17]. Properties and parameters of these amorphous structures are considered in comprehensive review [18].

Analysis of the existing scientific literature leads to the conclusion that the current study of amorphous ice passes only the accumulation phase of information without theoretical understanding. In many respects the results are determined by methods of sample preparation, modes of experimentation and applied research methods. Only indisputable conclusion can be made from the large number of reviews about the different states of water ice: by heating all existing crystalline and amorphous forms of water ice pass sequentially under normal pressure to metastable ice $I$, and then to the stable state ice $I_{b}$.

Nonequilibrium state and slow structural relaxation time is one of the main difficulties in the studying of amorphous ice. The structures of amorphous ice can not be determined by crystallographic space groups, as they are metastable with respect to the thermodynamically stable crystalline polymorphs. However, the construction of the radial distribution function, which in the literature is referred to as $R D F$ (radial distribution function) is used as a powerful method of investigation of amorphous structures. Practically $R D F$ is the Fourier transform of diffraction pattern, obtained, for example, by $X$-ray diffraction, and allows to determine the number of molecules at any distance from the molecule selected as the central $[3,19]$. For $\mathrm{H}_{2} \mathrm{O}$ the correlation of atomic pair oxygen-oxygen is calculated and atomic scattering factor of hydrogen is very small.

The first analysis of the amorphous structure with $R D F$ is made in [20]. Slow condensation of water vapor $(\sim 4 m g / h o u r)$ on a carefully polished copper substrate area of $2 \times 2 \mathrm{~cm}^{2}$ at a temperature of $77 \mathrm{~K}$ was performed on the $X$-ray diffractometer, designed to explore the non-crystalline materials. The measurement was carried out in two weeks, when the investigated sample reached a thickness of about $1 \mathrm{~mm}$.

Fig. 2 shows the pair correlation function $h_{\text {oo }}$ (r) of oxygen atoms calculated from diffraction patterns for liquid water [20], for polycrystalline hexagonal ice and $A S W$ [21]. Analysis of the data shows that the interaction between oxygen atoms extends only to a few molecular shells. The first peak, which characterizes the immediate surroundings can be described by a Gaussian distribution centered at $2.76 \AA$ with a standard deviation $0.1 \AA$. The area under this peak corresponds to the interaction of the four pairs of the oxygen atoms. Comparing $R D F$ specifies the undoubted differences $A S W$ structures and polycrystalline phase of normal pressure $I_{b}$. Moreover, the polycrystalline phase 

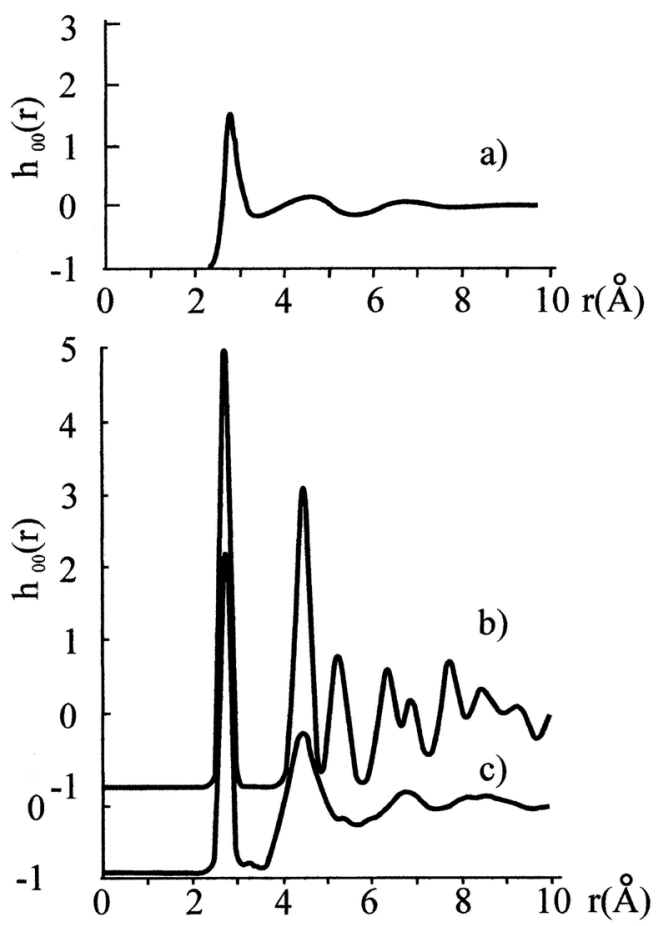

Fig. 2. The pair correlation functions of oxygen atoms: a) water at $3^{\circ} \mathrm{C}$; b) polycrystalline ice $I_{h}$ at $77 \mathrm{~K}$; c) $A S W$ at $77 \mathrm{~K}$.

has a density of $0.93 \mathrm{~g} \cdot \mathrm{cm}^{-3}$ at normal pressure, and density estimation of amorphous ice for initial slope of $R D F$ is about $1.2 \mathrm{~g} \cdot \mathrm{cm}^{-3}$. Thus, under normal pressure $R D F$ for liquid water and ASW are much like, however, the maxima $\mathrm{RDF}$ for liquid water slightly shifted on longer distances and broadened as compared with the maxima for $A S W$.

\section{AMORPHOUS ICE AT LOW TEMPERATURES AND HIGH PRESSURES}

In 1984, for the first time from hexagonal ice at a temperature of $77 \mathrm{~K}$ and a pressure of 1 $G P a$, high-density amorphous ice HDA (highdensity amorphous) obtained by "melting" the crystalline hexagonal ice below the glass transition temperature $T_{g}$ [22]. The presence of amorphous phase ice was confirmed by $X$-ray powder diffraction. The density of the obtained sample was $1.31 \mathrm{~g} / \mathrm{cm}^{3}$ when a pressure of $1 \mathrm{GPa}$ and $1.17 \mathrm{~g} / \mathrm{cm}^{3}$ at standard pressure. Subsequent annealing of the sample at atmospheric pressure led to the formation of a new amorphous phase with a density of $0.94 \mathrm{~g} / \mathrm{cm}^{3}$. This phase is indicated in the literature as $L D A$ (low-density amorphous) ice. Measuring by $X$-ray and neutron diffraction methods indicate the identity of structures for $L D A, A S W$ and $H G W$ [23]. Small differences of the thermodynamic properties of these structures is likely due to differences in the methods of preparation of material samples and experimental procedures. Currently, there is a single point of view that the $L D A, A S W$ and $H G W$ are one and the same material [19]. The work [22] marked the beginning a new line of research of amorphous ice and their mutual transformations at high pressures (about $1 \mathrm{GPa}$ ) and low temperatures (below 77K). As example of a rich polyamorphism water ice, that is the existence of more than one amorphous state, was the discovery of a new type of amorphous ice, called VHDA (very high density amorphous) ice [24]. Amorphous ice $V H D A$ was received isobaric heating $H D A$ from $77 \mathrm{~K}$ to $165 \mathrm{~K}$ at a pressure of $1.1 \mathrm{GPa}$ and subsequent reduction to normal pressure at a temperature of $77 \mathrm{~K}$. VHDA density was found to be $1.25 \pm 0.01 \mathrm{~g} /$ $\mathrm{cm}^{3}$, which is $\sim 9 \%$ higher than the density of $H D A$. VHDA turns back to HDA during isochoric heating to $140 \mathrm{~K}$ at an initial pressure of $0.02 \mathrm{GPa}$ or to $L D A$ in the isobaric heating. Polyamorphism has now been observed in many other substances such as $\mathrm{SiO}_{2}, \mathrm{GeO}_{2}, \mathrm{Si}, \mathrm{Ge}$ [25]. However, aqueous polyamorphism is so unique that it appears there is no substance capable of competing with water in a manifold of known forms. Numerous conversion between amorphous states of water ice and his various stable and metastable crystalline states in detail are considered in the last comprehensive review [26].

After the release of seminal paper [7] it was believed that only two forms of high and low density, i.e. $L D A$ and $H D A$, can exist among all amorphous forms of water ice. However, the existence of the intermediate amorphous 
forms of the mutual conversion of $L D A$ and $H D A$ was shown in [27] using the methods of neutron and $X$-ray diffraction. For structure comparison, samples of $H D A$, derived from Ih at a temperature of $77 \mathrm{~K}$ and pressure $18 \mathrm{kbar}$, annealed to temperatures of $95 \mathrm{~K}, 100 \mathrm{~K}, 105 \mathrm{~K}$, $110 K$ (up until the $L D A$ ) and reduced to $40 K$. Constructed by the authors from the diffraction patterns $R D F$ function are shown in Fig. 3.

As the temperature changes the position of the first peak, which determines the distance of interaction of pairs of oxygen through hydrogen bonding, regularly changes from $2.80 \AA$ to $2.76 \AA$ for $H D A$ for the phase with the lowest density. There are main

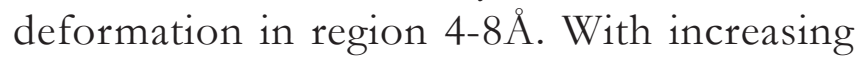
annealing temperature the broad peak $(\sim 6.4 \AA)$ is shifted towards longer distances and intensity of the broadened peak $(\sim 4.5 \AA)$ increases. These results indicate the possible existence of a continuous intermediate metastable phases and extremely complex structural transformation of amorphous forms of ice obtained at high pressures and low temperatures.

Thus, at the present time the polyamorphism presence of water ice at low temperatures is not contested and this significantly expands our knowledge of the phase states of water. Modern simplified diagram of the temperaturepressure water to the different phases is presented in Fig. 4 [28]. A feature of the chart is the fact that, since the normal pressure, the

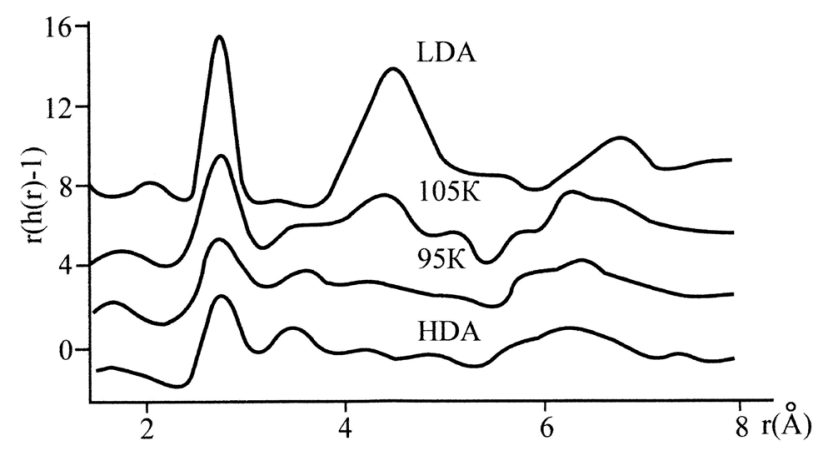

Fig. 3. $R D F$ for $H D A$, annealed at various temperatures up to $L D A$.

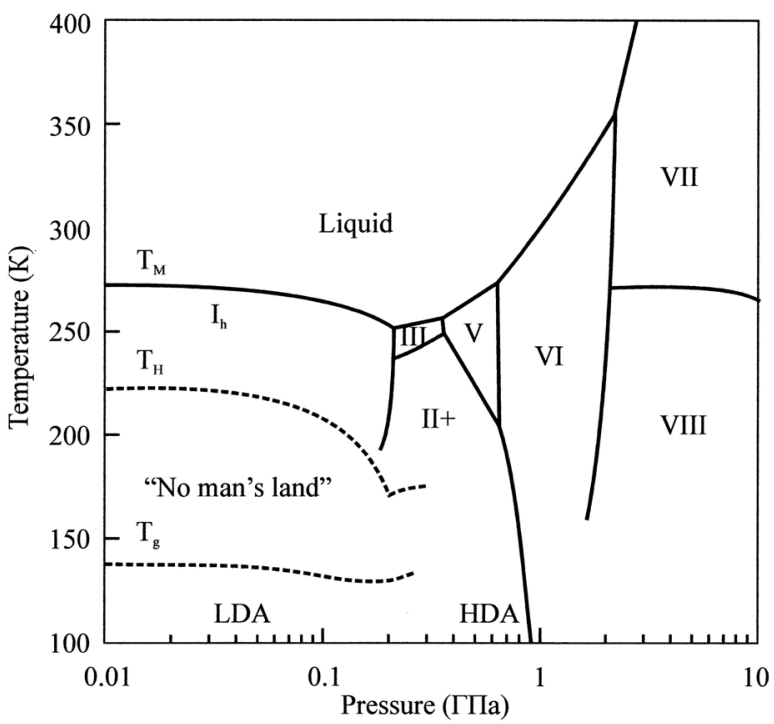

Fig. 4. The phase diagram of water.

melting line $T_{M}$ drops to the temperature $251 \mathrm{~K}$ at a pressure of $0.2 \mathrm{GPa}$, and then rises to a high temperature equidistant from line of the homogeneous nucleation $T_{H}$ when the pressure increases. Line homogeneous nucleation $T_{H}$, situated below the melting line $T_{M}$, denotes a lower temperature at which the freezing water can exist without crystallization. Below this line the volumetric mass of water can not exist. The glass transition line $T_{g}$ determines the highest temperature at which an amorphous ice can exist without crystallization. Several different types of ice included in the chart area, designated II+. Upon application of a high pressure of about $1 \mathrm{GPa}$ and temperatures below $150 \mathrm{~K}$ the crystalline ice Ih transforms to the amorphous ice $H D A$, which in turn, if the pressure is removed, spontaneously becomes amorphous ice type $L D A$. Annealing $H D A$ to $120 \mathrm{~K}$ at high pressure leads to a more dense amorphous ice type $\operatorname{VHD} A$, which, when the pressure is removed, transforms to the $L D A$ also.

It should be noted that given the modern phase diagram of water confirms the complete lack of published data on the presence of amorphous ice near the melting point at atmospheric pressure. 


\section{THE AMORPHOUS ICE NEAR THE MELTING POINT AT ATMOSPHERIC PRESSURE}

To study diffuse $X$-ray scattering by ice at temperatures close to its melting point, we developed a precise low-temperature semiautomatic attachment to $X$-ray diffractometer [29]. The attachment is made on the basis of the Peltier element. This attachment provides adjustment and maintaining the temperature of the test sample in the range from $0^{\circ} \mathrm{C}$ to $-25^{\circ} \mathrm{C}$ with an accuracy of $\pm 0.1^{\circ} \mathrm{C}$ for multi-day recording of diffraction patterns. $25 \mathrm{~W}$ Peltier element freezes the copper cuvette by size of $30 \times 30 \times 10 \mathrm{~mm}^{3}$ with a volume of the test sample of $1.4 \mathrm{~cm}^{3}$. Due to the small size of the cell determined by the geometry of the $X$-ray diffractometer, efficient removal of significant amounts of heat from the Peltier element was performed using a copper chamber with volume of $4.5 \mathrm{~cm}^{3}$, through which coolant was pumped. Measuring the temperature of the sample was performed $K$-type thermocouple attached to the body of the copper cuvette.

Measuring the intensity of the diffuse scattering of $X$-rays was performed on an automated $X$-ray diffractometer type DRON on $\mathrm{CuK}_{\alpha}$ radiation. Monochromatization of beam is provided by a flat single crystal silicon (reflecting plane (111)). The scattered radiation was detected using a scintillation counter BDS6-05. The sample, counter and crystal of the monochromator were located on one of the focusing circle. To ensure the independence of the absorption coefficient on the angle of incidence of $X$-rays on the sample survey was used, in which the angle of incidence of $X$-rays on the sample was equal to the angle of reflection. To obtain minimum air $X$-ray scattering collimators were used by which eliminates most of the scattering by air. The remainder of the radiation scattered by the air that enters to the counter, was excluded by subtracting of half intensity measured in the absence of the sample. The intensity of the diffuse scattering consists of two parts: 1)own diffuse scattering, 2)"parasitic" diffuse background, which includes: a)cosmic background and the noice of equipment, b) cattering of the air during the passage of the X-ray beam from the tube to the counter, c)Compton scattering. The cosmic background and noise of equipment were determined when a high voltage is switched off. All of the components of the "parasitic" diffuse background were excluded from the measured values of intensities.

The intensities of the scattered $X$-ray radiation were leaded to the electronic units by measuring at large angles of scattering intensity of fused quartz according to [30]. The results of measurements of the scattering intensity of $X$-rays with ice prepared from distilled water, conducted at a temperature of $-10^{\circ} \mathrm{C}$ are shown in Fig. 5. It is seen that in the diffractogram in accordance with the phase diagram of the ice there are the structural reflexes, which are limited on level 1000 pulses. The positions of structural reflexes correspond to hexagonal crystalline ice lattice with parameters $a=4.52 \AA$ and $c=7.36 \AA$ [31]. As is well known, the intensity of diffuse scattering by several orders of magnitude less than the intensity of the strongest reflexes, therefore often the diffuse scattering is excluded from consideration. To prove the existence of diffuse scattering is necessary to use highly sensitive diffractometer. It is seen that the diffraction pattern of hexagonal crystalline ice

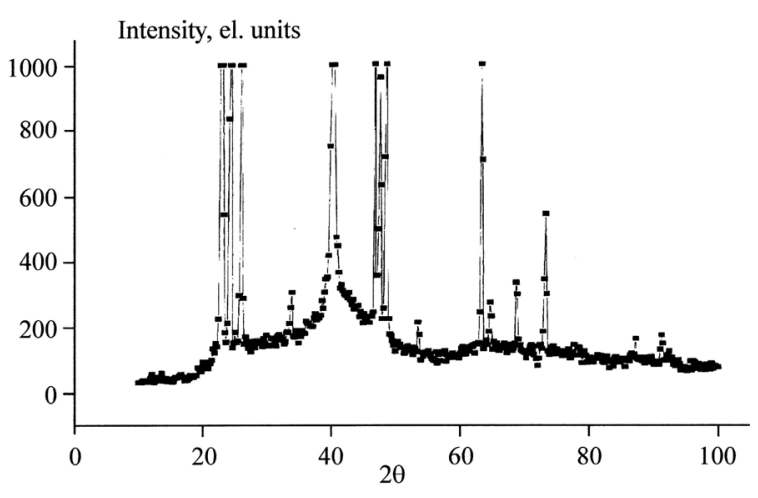

Fig. 5. The XRD pattern of ice, obtained at $-10^{\circ} \mathrm{C}$. 
indicates an intense $X$-ray diffuse scattering, as evidence of the existence in the sample the proportion of non-crystalline phase. The study of such diffuse scattering in metals and metal alloys provides the valuable information on the imperfections of their crystal structure, and about the short-range order and static displacements [30, 32].

Also, the study was conducted the scattering of $X$-rays near the melting point of ice. Fig. 6 shows the results of measurements carried out at a temperature of $-3^{\circ} \mathrm{C}$ [33]. It is seen that in the diffraction pattern of ice, obtained near the melting point, there is no structural reflections of hexagonal ice, but there is intense diffuse scattering that is typical for the amorphous state. Ibidem there are traces of almost completely destroyed of the crystal structure of $I_{b}$ ice. The maximum value of the diffuse scattering of ice corresponds to the angle of sliding $2 \Theta$, equal to $22.3^{\circ}$.

For the purpose of constructing the radial distribution function $F(r)=4 \pi r^{2} \varrho(r)$ of the metastable amorphous ice was used [8]

$$
4 \pi r^{2} \rho(r)=4 \pi r^{2} \rho_{0}+\frac{2 r}{\pi} \int_{0}^{\infty} s i(s) \sin r s d s,
$$

where $r$ - the distance from the center of every atom of oxygen, $\varrho_{0}-$ density of water or ice, expressed in molecules per cubic angstroms, $s=$ $(4 \pi \sin \Theta) / \lambda, i(s)=I / N f-1, f-$ atomic scattering factor, $I / N$ - experimentally measured intensity, expressed in electron units.

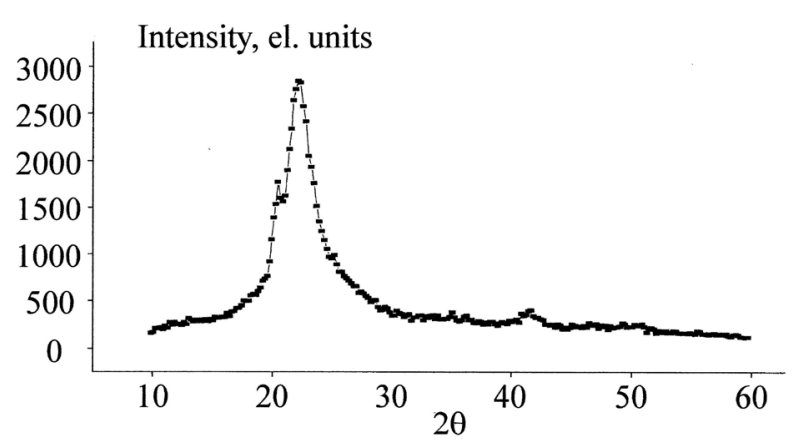

Fig. 6. The XRD pattern of ice, obtained near the melting point at atmospheric pressure.

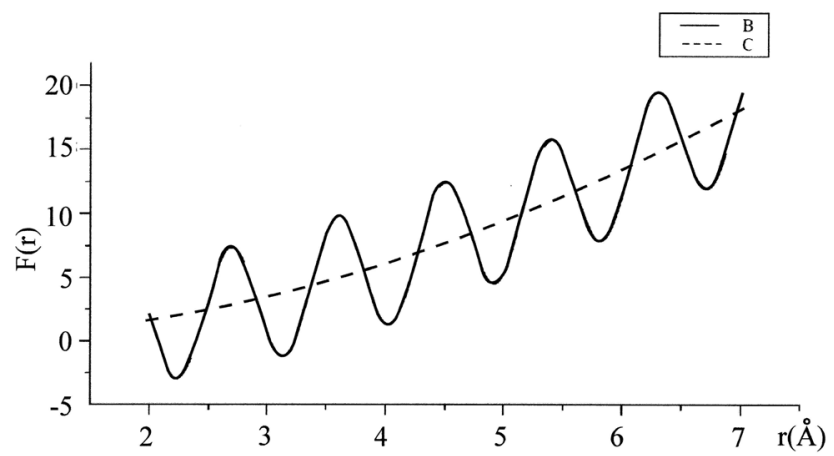

Fig. 7. The dependence of the radial distribution function $F(r)$ of amorphous ice from interatomic distance $r:\left(4 \pi r^{2} \mathrm{Q}(r)\right.$ - curve B; $4 \pi r^{2} \varrho_{0}-$ curve $C$ ).

Equation (1) can be used to construct a radial distribution function of the amorphous ice in the assumption that the contribution of the hydrogen atoms in the intensity of $X$-ray scattering is very small.

Calculation of the radial distribution function of the amorphous ice $4 \pi r^{2} \varrho(r)$ was held in the range of $r$ from $2 \AA$ to $7 \AA$. The calculation results are shown in Fig. 7. From Fig. 7 it is seen that the radial distribution function of the amorphous ice $4 \pi r^{2} \varrho(r)$ is an oscillating function (curve $B$ ) around a smooth function $4 \pi r^{2} \varrho_{0}$ (curve $C$ ). An alternation of maxima and minima of the curve is preserved for large $r$. However, with the growth of the interatomic distance $r$ there are deviations from periodicity which is typical of the crystalline state. The provisions of the first five maxima of the function $4 \pi r^{2} \varrho(r)$ amorphous ice proved to be equal to $2.7 \AA, 3.6 \AA, 4.5 \AA, 5.4 \AA$ and $6.3 \AA$ accordingly. The observed maxima of this function characterizes the position of the maximum values of the electron density distribution in a sample of metastable amorphous ice and should correlate with the radial distribution of oxygen atoms in the crystal lattice of $I_{b}$ ice. The arrangement of atoms of hydrogen and oxygen in the crystal lattice of hexagonal ice is shown in Fig. 8. Based on the layout of the oxygen atoms in the crystal lattice of ice $I_{b}$ (Fig. 8), it was 


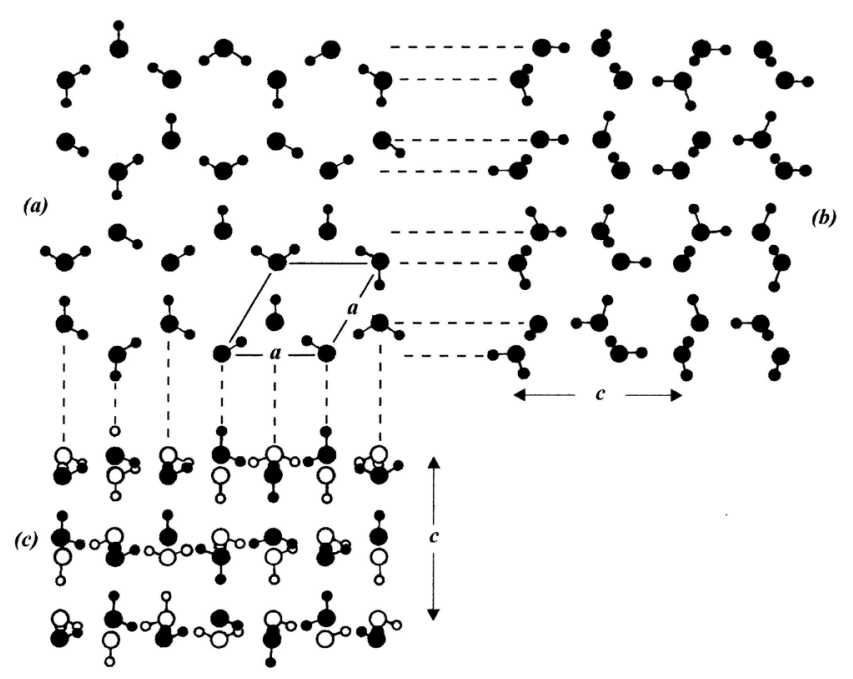

Fig. 8. Location oxygen and hydrogen atoms in the crystal lattice of hexagonal ice. The oxygen atoms hying at different levels, showing large and bydrogen atoms - small circles $(a=$ 4.52 A, c = 7.36 A) [31]. Projections along /001/ (a), $/ 100 /(b), / 120 /(c)$.

calculated the radii of the first five of the coordination spheres. They turned out to be equal to $2.61 \AA, 3.69 \AA, 4.52 \AA, 5.22 \AA$ and $6.9 \AA$ accordingly. Comparing them with the values of the first five positions of the maxima of the curve of calculated radial distribution function of the metastable amorphous ice we see that for the first four focal areas they were close in value. The greatest discrepancy is observed for the fifth coordination sphere. It was about $10 \%$. Such consent is not random and indicates on quasicrystal structure of metastable amorphous ice that occurs near the melting point. As shown by numerous experiments, the use of equipment with a copper cold finger and small Dewar with liquid nitrogen was not allowed smoothly rearrange the sample temperature during the experiment and require considerable effort to maintain the desired temperature. Therefore, we developed a precision semiautomatic device for maintaining the temperature of the test sample for $X$-ray diffractometer [34]. The basis of the device is a thermoelectric converter based on the Peltier element with power $25 \mathrm{~W}$ which provides stable and smooth control of the sample temperature. As shown

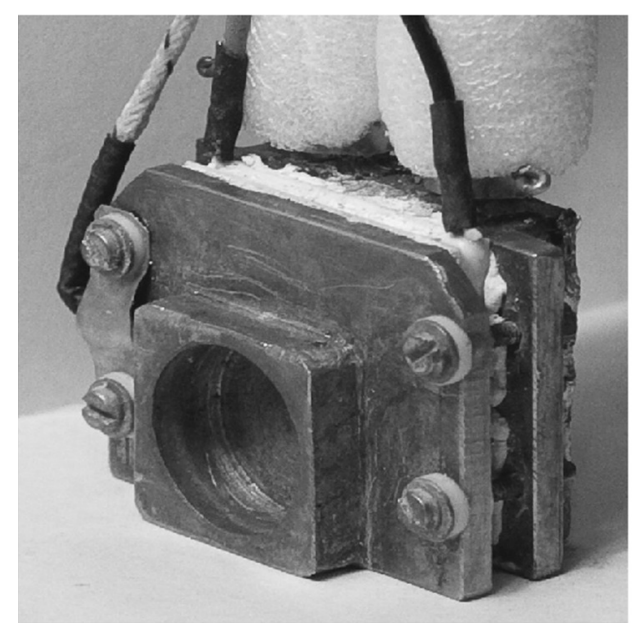

Fig. 9. The cuvette with the thermoelectric converter.

in Fig. 9 Peltier element is sandwiched between copper cuvette with the sample and the copper cooling chamber through a layer of thermal paste KPT-8 which retains its properties over a wide temperature range. The copper cuvette has dimensions $30 \times 30 \times 10 \mathrm{~mm}^{3}$, the test sample volume of $1.4 \mathrm{~cm}^{3}$. The main difficulty in creating such device is to take heat from the Peltier element size of $30 \times 30 \times 4 \mathrm{~mm}^{3}$ at small size of the device defined by the geometry of the $X$-ray diffractometer. To remove heat from the hot side of element the pump through hoses pumps over the antifreeze with freezing point $-40^{\circ} \mathrm{C}$ through the attached cooling copper chamber of volume of $4.5 \mathrm{~cm}^{3}$. In turn, antifreeze was cooled in a 4 liter vessel installed in the freezer providing temperature of antifreeze up to $-25^{\circ} \mathrm{C}$. The measurement the sample temperature was carried out by $K$-type thermocouple attached to the body of copper cell. The developed device can finely to adjust and to maintain temperature of the investigated sample in the range from $0^{\circ} \mathrm{C}$ to $-25^{\circ} \mathrm{C}$ with an accuracy of $\pm 0.1^{\circ} \mathrm{C}$ for many hours shooting diffractogram. Measurements of the intensity the diffuse scattering of the $X$-rays were performed on automated $X$-ray diffractometer type $D R O N$ on $C u K_{\alpha}$ radiation, as described above. For calculating of the radial distribution function was used the expression (1). 
On Fig. 10 is shown a diffraction pattern obtained at a temperature of ice at $-10^{\circ} \mathrm{C}$ [35]. It is seen that, depending the intensity of the diffuse $X$-ray scattering from angle $2 \theta$, reduced after eliminating intense reflections of structural phase $I_{b}$, there is a diffuse maximum in the vicinity of $40^{\circ}$, indicating a significant proportion of non-crystalline phase.

To identify the nature of the diffuse maximum after exclusion of spurious components, and subtracting the absolute Compton scattering the radial distribution function is calculated in the range for $r$ from $2 \AA$ to $7 \AA$ and is shown in Fig. 11. It can be seen that the curve $F(r)$ oscillates around a smooth curve function $4 \pi r^{2} \varrho_{0}$ (curve C). Such oscillations indicate the oscillations of the electron density of the amorphous portions of ice. It is also seen that in contrast to the results presented in [34], the first maximum of a fully amorphous ice, which was located near the $r \approx 2.7 \AA$, splits into two peaks at $r \approx 2.6 \AA$ and $r \approx 3.1 \AA$. Such splitting can be interpreted using the ice crystal structure model [31], given also in [33] in Fig. 6. Analysis of the structure of amorphous ice [33] showed that the contribution in the first maximum of the curve $F(r)$ of the completely amorphous ice are making both the oxygen atoms, which are located in the corners

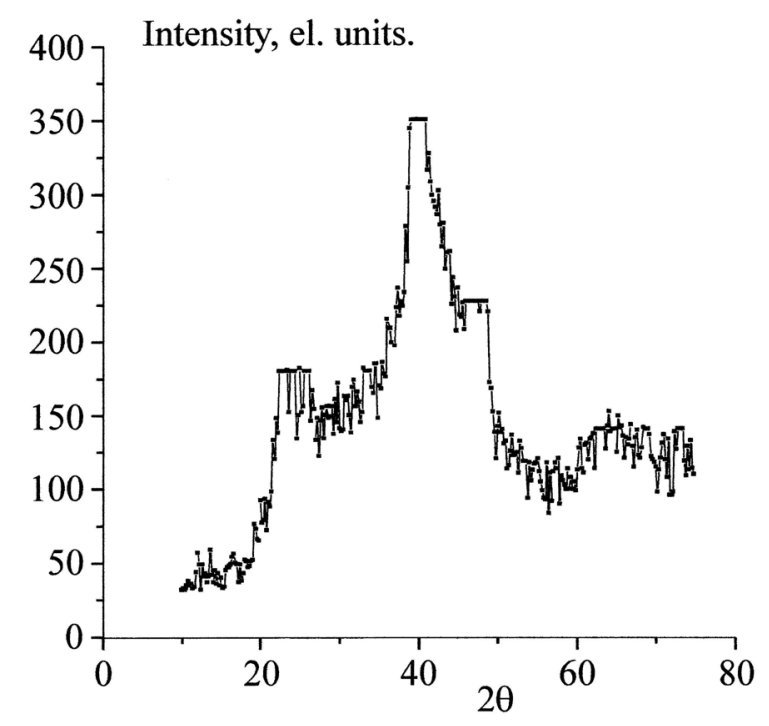

Fig. 10. The XRD pattern of ice prepared from distilled water, obtained at $-10^{\circ} \mathrm{C}$.

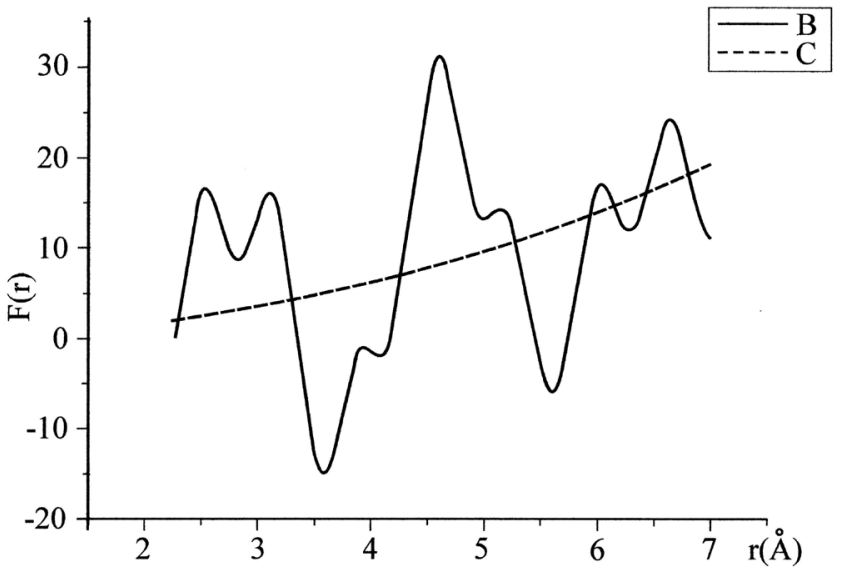

Fig. 11. The dependence of the radial distribution function $F(r)$ of amorphous ice fraction of the interatomic distance $r$ for XRD pattern, obtained at $-10^{\circ} \mathrm{C}:\left(4 \pi r^{2} \mathrm{Q}(r)-\right.$ curve $\mathrm{B}$;

$$
\left.4 \pi r^{2} Q_{0}-\text { curve } C\right) \text {. }
$$

of hexagons, and the oxygen atoms, which are located at the nearest distance but on different levels. In the presence of structural reflections in the diffraction pattern of the sample of the ice the structure of amorphous ice is different from the ideal. In particular, it is probably distorted by increasing the interatomic distances between the oxygen atoms lying at different levels. This can condition the splitting of the first maximum of the radial distribution function in samples of ice with the non-ideal amorphous structure.

Fig. 12 shows a diffraction pattern obtained at the ice temperature $-3^{\circ} \mathrm{C}$. It can be seen

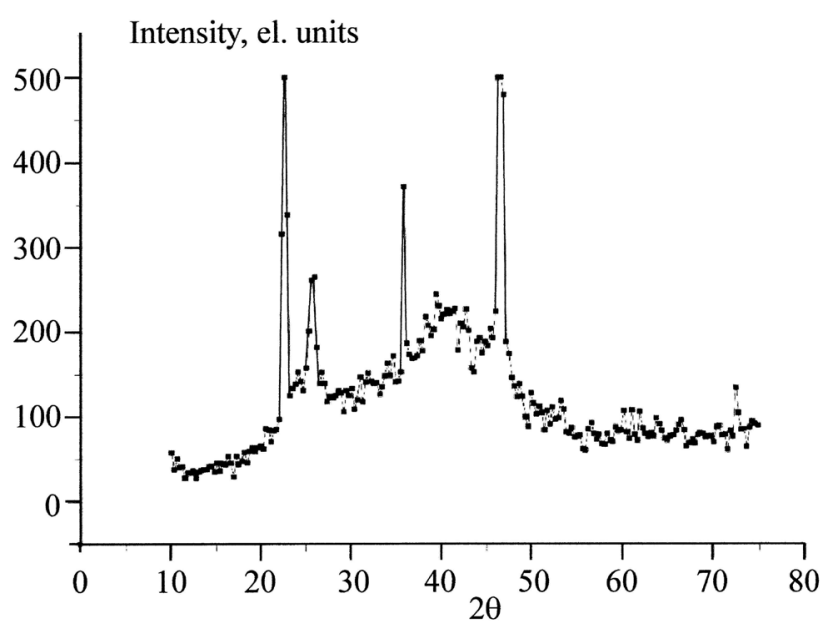

Fig. 12. The XRD pattern of ice prepared from distilled water, obtained at $-3^{\circ} \mathrm{C}$. 
that, unlike the previous case, depending on the intensity of the diffuse $X$-ray scattering from angle $2 \theta$ there are weak reflexes of phase Ih and an intense modulated diffuse scattering with diffuse maximum, indicating a significant proportion of non-crystalline phase. The radial distribution function, based on this diffraction pattern is shown in Fig. 13. As in the previous case, the curve $F(r)$ oscillates around a smooth curve function $4 \pi r^{2} \varrho_{0}$ (curve $C$ ), indicating on the oscillations of the electron density of the amorphous ice. The curve $F(r)$ at $r \approx 2.6 \AA$ and $r$ $\approx 3.0 \AA$ indicate two peaks rather than one that is typical for the ideal structure of amorphous ice [33] (in the absence of reflexes of the crystalline phase). Such splitting also corresponds to an increase of the interatomic distances between the oxygen atoms lying at different levels. Subsequent maxima of radial distribution function $F(r)$ also correspond to model presented in [31].

These results suggest strongly presence of the amorphous phase near the melting point of ice. However, the question arises about the temporal stability of the amorphous portion of ice and the possibility of reaching an equilibrium state of the system in the presence of polycrystalline and amorphous components.

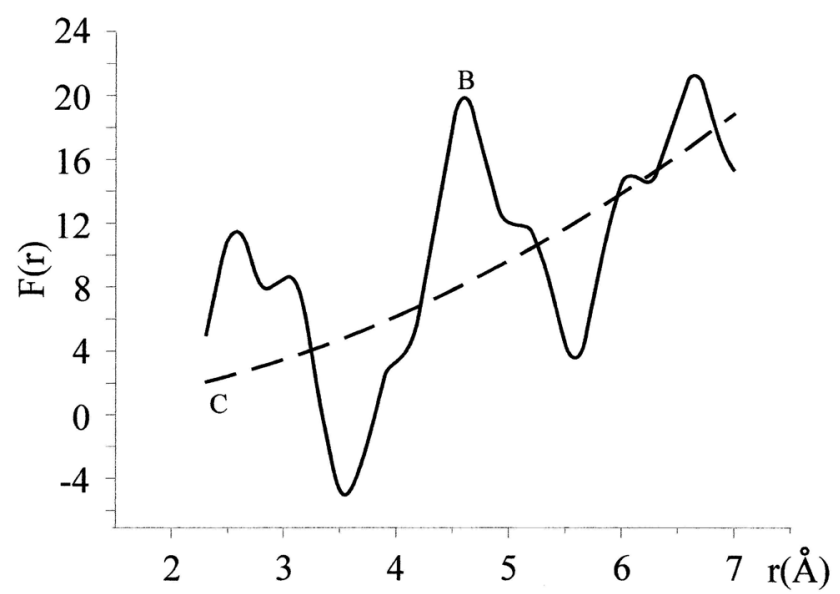

Fig. 13. The dependence of the radial distribution function $F(r)$ of amorphous ice fraction of the interatomic distance $r$ for XRD pattern, obtained at $-3^{\circ} C:\left(4 \pi r^{2} \varrho(r)-\right.$ curve $B$; $4 \pi r^{2} \varrho_{0}-$ curve $\left.C\right)$.
To answer this question for two weeks one and the same sample of ice made from distilled water is constantly maintained at a temperature of $-10^{\circ} \mathrm{C}$ and investigated on a diffractometer at regular intervals.

Fig. 14 shows some of the diffraction patterns obtained during a two-week registration sample of ice at $-10^{\circ} \mathrm{C}$. It is evident that diffuse maxima observed at angles of $40^{\circ}$ and $70^{\circ}$ on $2 \theta$ gradually disappear and redistributed to $24^{\circ}$ on $2 \theta$, practically maintaining the value of the scattering intensity. On some individual diffraction patterns there are intensive reflections

300 Intensity, el. units.

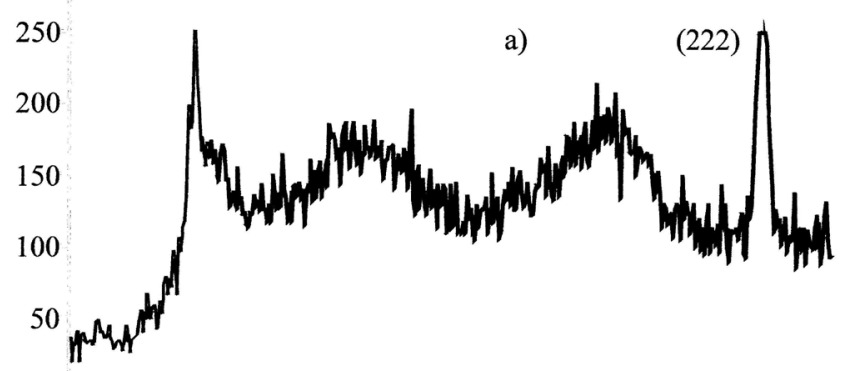

300 Intensity, el. units.
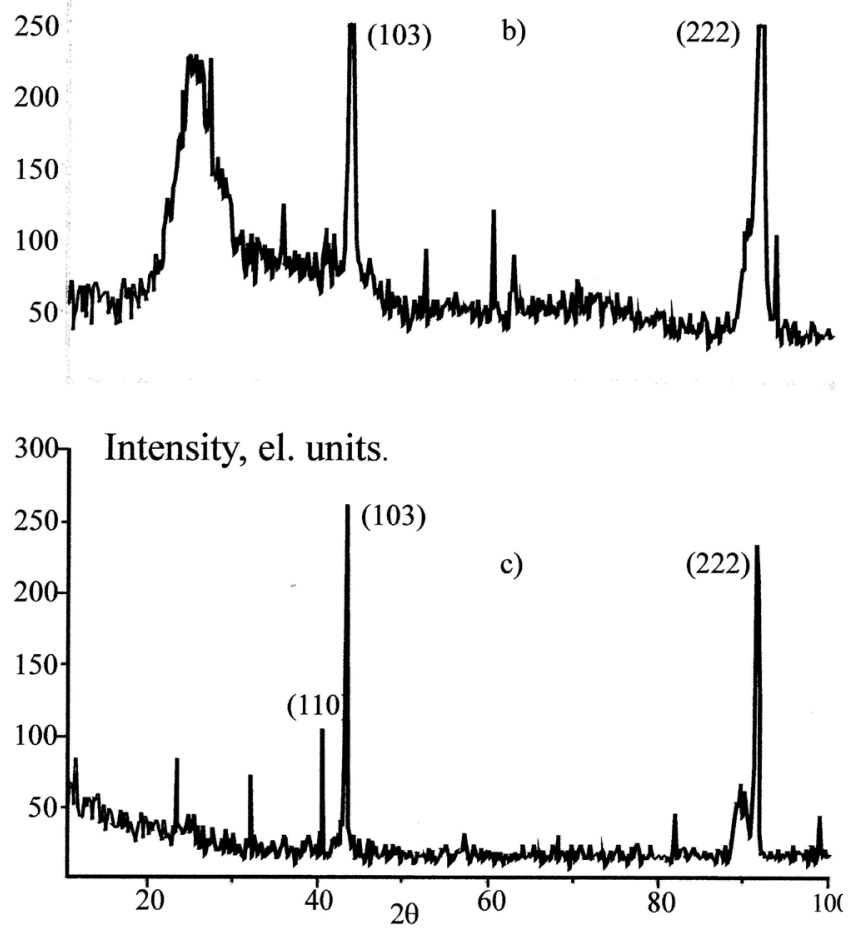

Fig. 14. The diffraction patterns obtained from distilled water ice at a temperature of $-10^{\circ} \mathrm{C}:$ a) on the 6 th day; b) on the 12th day; c) on the 15 th day of registration. 
of hexagonal ice, which can be explained by best position for recording of single crystals of Ih ice which are formed. On the 15 th day, there is a complete disappearance of the diffuse scattering of the radiation on ice up to the level of residual $X$-ray scattering in air. Evidently, there is the complete formation of the crystalline phase of the sample. Apparently, the absence of other reflexes is determined by a non-optimal arrangement of single crystals of hexagonal ice.

\section{CONCLUSION}

On the diffraction patterns of ice prepared from distilled water along with the reflexes hexagonal ice there are the diffuse $X$-ray scattering related to disordered phases ice. On one of the diffraction patterns of ice in the almost complete absence of structural reflexes there is the diffuse maximum which is typical for the amorphous state. The studies demonstrated coexistence under normal pressure the amorphous structure and a crystalline structure of hexagonal ice near its melting point. A permanent change in the character of diffuse scattering with a continuous redistribution of the intensity maxima from one to another was revealed on the diffraction patterns of the sample ice, supported a long time at a constant temperature of $-10^{\circ} \mathrm{C}$. We proved the metastable character of amorphous phase of ice, derived from distilled water. In the future, there is necessary studying the static and dynamic structural deformations of ice at a temperature close to the phase transition temperature.

\section{REFERENCES}

1. Hobbs PV. Ice physics. Oxford, Clarendon press, 1974, $837 \mathrm{p}$.

2. Salzmann CG, Radaelli PG, Hallbrucker A, Mayer E and Finney JL. The Preparation and Structures of Hydrogen Ordered Phases of Ice. Science, 2006, 311:1758-1761.

3. Bernal JD, Fowler RH. A Theory of Water and Ionic Solution, with Particular Reference to Hydrogen and Hydroxyl Ions. J. Chem. Phys. 1933, 1:515-548.
4. Zheligovskaya EA, Malenkov CG. Crystalline water ice. Russian Chem. Rev, 2006, 75(1):57-76.

5. Angell CA, Shuppert J, Tucker JC. Anomalous properties of supercooled water. Heat capacity, expansivity, and proton magnetic resonance chemical shift from 0 to $-38 \%$. J. Phys. Chem., 1973, 77:3092.

6. Elsaesser MS, Winkel K, Mayer E, Loerting T. Reversibility and isotope effect of the calorimetric glass $\rightarrow$ liquid transition of low-density amorphous ice. Phys. Chem. Chem. Phys., 2010, 12:708.

7. Mishima O., Stanley E. The relationship between liquid, supercooled and glassy water. Nature, 1998, 396(26 November):329.

8. Morgan J, Warren BE. X-ray Analysis of the Structure of Water. J.Chem.Phys., 1938, 6:666.

9. Dowell LG, RinfretAP. Low-temperature forms of ice as studied by X-ray diffraction. Nature, 1960, 188:1144-1148.

10. Salzmann CG, Kohl I, Loerting T, Mayer E, Hallbrucker A. The low-temperature dynamics of recovered ice XII as studied bydifferential scanning calorimetry: a comparison with ice V. Phys. Chem. Chem. Phys., 2003, 5:3507.

11. Burton EF, Oliver WF. The Crystal Structure of Ice at Low Temperatures. Proc. R. Soc., 1935, A153:166-172.

12. Shallcross FV, Carpenter GB. X-ray Diffraction Study of the Cubic Phase of Ice. J. Chem. Phys., 1957, 26:782-784.

13. Beaumont RH, Chihara H, Morrison JA. Transitions between Different Forms of Ice. J. Chem. Phys., 1961, 34:1456-1457.

14. Olander DS, Rice SA. Preparation of Amorphous Solid Water. Proc. Nat. Acad.Sci., US A, 1972, 69(1):98-100.

15. Mayer E, Bruggeller P. Vitrification of pure liquid water by high pressure jet freezing. Nature, 1982, 298(19):715-718.

16. Jenniskens P, Banham SF, Blake DF, McCoustra MRS. Liquid water in the domain of cubic crystalline ice $I_{c}$ J.Chem.Phys., 1997, 107:1232. 
17. Bowron DT, Finney JL, Hallbrucker A, Kohl I, Loerting T, Mayer E, Soper AK. The local and intermediate range structures of the five amorphous ices at $80 \mathrm{~K}$ and ambient pressure: A Faber-Ziman and Bhatia-Thornton analysis. J. Chem. Phys., 2006, 125:194502.

18. Debenedetti PG. Supercooled and glassy water. J. Phys.: Condens. Matter, 2003, 15:R1669-R1726.

19. Katzoff S. X-ray Studies of the Molecular Arrangement in Liquids. J.Chem.Phys., 1934, 2:841.

20. Venkatesh CG, Rice SA, Narten AH. Amorphous Solid Water: An X-ray Diffraction Study. Science, 1974, 186:927-928.

21. Narten AH, Venkatesh CG, Rice SA. Diffraction pattern and structure of amorphous solid water at 10 and $77 \mathrm{~K}$. J. Chem. Phys., 1976, 64(3):1106-1121.

22. Mishima O, CalvertLD, Whalley E. 'Melting ice' $\mathrm{I}$ at $77 \mathrm{~K}$ and $10 \mathrm{kbar}$ a new method of making amorphous solids. Nature, 1984, 310:393.

23. Bellissent-Funel M-C, Bosio L, Hallbrucker A, Mayer E, Sridi-Dorbez R. X-ray and neutron scattering studies of the structure of hyperquenched glassy water. J. Chem. Phys., 1992, 97:1282.

24. Loerting T, Salzmann C, Kohl I, Mayer E, Hallbrucker A. A second distinct structural "state" of high-density amorphous ice at $77 \mathrm{~K}$ and 1 bar. Phys. Chem. Chem. Phys., 2001, 3:5355.

25. Loerting T, Giovambattista N. Amorphous ices: experiments and numerical simulations. J. Phys.: Condens. Matter, 2006, 18:R919-R977.

26. Loerting T, Winkel K, Seidl M, Bauer M, Mitterdorfer C, Handle PH, Salzmann CG, Mayer E, Finney JL, Bowron DT. How many amorphous ices are there? J. Phys. Chem. Chem. Phys., 2011, 13:8783-8794.

27. Tulk CA, Benmore CJ, Urguidi J, Klug DD, Neuefeind J, Tomberli B, Egelstaff PA. Structural Studies of Several Distinct Metastable Forms of Amorphous Ice. Science, 2002, 297(5585):1320-1323.

28. Soper AK. Structural transformations in amorphous ice and supercooled water and their relevance to the phase diagram of water. J. Molecular Physics, 2010, 106:1-36.

29. Silinov VM, Chubarov VV. Nizkotemperaturnaya pristavka k rentgenovskomu difraktometru dlya izmereniya intensivnosti rasseyaniya rentgenovskikh luchey zamorozhennymi zhidkostyami [Low-temperature attachment to the diffractometer $\mathrm{x}$-ray to measure the intensity of x-ray scattering of frozen liquids]. Preprint №11 MGU, Fizicheskiy fakultet. Moscow, MGU Publ., 2008, 7 p. (in Russ.).

30. Iveronova BI, Katsnelson AA. Bliz̧hniy poryadok $v$ tverdylkh rastvorakh [Short-range order in solid solutions]. Moscow, Nauka Publ., 1977, 250 p.

31. Lonsdale DK. The Structure of Ice. Proc. Royl. Soc., 1958, 247:424-434.

32. Krivoglaz MA. Diffuznoe rasseyanie rentgenovskikh luchey $i$ neytronov na fluktuatsionnykh neodnorodnostyakh v neideal'nykh kristallakh [Diffuse $\mathrm{X}$-ray and neutrons scatterings on fluctuational inhomogenities in nonideal crystals]. Kiev, Naukova dumka Publ., 1984, 288 p.

33. Silonov VM, Chubarov VV. Diffuznoe rasseyanie rentgenovskikh luchey l'dom vblizi tochki plavleniya [Diffuse X-ray scattering by ice near melting point]. Vestnik MGU, Fizika, Astronomija, 2011, 4:48-52 (in Russ.).

34. Silonov VM, Chubarov VV. Pretsizionnaya nizkotemperaturnaya pristavka $\mathrm{k}$ rentgenovskomu difraktometru dlja izmerenija diffuznogo rasseyaniya rentgenovskikh luchey l'dom. [Precise lowtemperature device for $\mathrm{X}$-ray diffractometer for measuring of X-ray diffuse scattering by ice]. Preprint N2, MGU Fizicheskiy fakul'tet. Moscow, MGU Publ., 2013, 7 p. (in Russ.).

35. Silonov VM, Chubarov VV. Amorfizatsiya l'da vblizi tochki plavleniya [Amorphization of ice near the melting point]. Poverkhnost'. Rentgenovskie, sinkhrotronnye $i$ neytronnye issledovaniya, 2014, 5:1-5 (in Russ.). 